\title{
Determinantes socioeconômicos do diabetes mellitus em um contexto de desigualdades no nordeste brasileiro
}

\author{
Socioeconomic determinants of diabetes mellitus in a context of inequalities in northeast \\ Brazil
}

\section{Determinantes socioeconómicos de la diabetes mellitus en un contexto de desigualdades en el noreste de Brasil}

Silvia Pereira da Silva de Carvalho Melo ${ }^{1 *}$, Maria Nelly Sobreira de Carvalho Barreto ${ }^{1}$, Nathália Paula de Souza², Pedro Israel Cabral de Lira², Eduarda Ângela Pessoa Cesse ${ }^{1}$.

\section{RESUMO}

Objetivo: Analisar a prevalência e os determinantes socioeconômicos do diabetes mellitus em adultos no estado de Pernambuco, Nordeste brasileiro, em 2015/2016. Métodos: Trata-se de um estudo transversal, com uma amostra de 1019 adultos, baseado na IV Pesquisa Estadual de Saúde e Nutrição. A variável dependente foi definida considerando diabéticos aqueles com glicemia de jejum $\geq 126 \mathrm{mg} / \mathrm{dl}$ ou que referiram o seu diagnóstico durante entrevista. Analisou-se possíveis associações do diabetes com determinantes socioeconômicos, por meio de regressão multivariada de Poisson, considerando-se como significantes aqueles com valor de $p<0,05$. Resultados: A prevalência de diabetes foi de $10,8 \%$, sendo maior entre as pessoas com menor escolaridade (19\%), aposentados (23\%), que não recebiam bolsa família $(14,1 \%)$ e que apresentavam destino de dejetos $(15,9 \%)$ e abastecimento de água $(15,8 \%)$ ofertados de forma irregular. No modelo multivariado as variáveis socioeconômicas associadas ao diabetes foram: escolaridade, ocupação, bolsa família e abastecimento de água. Conclusão: O estudo revelou elevada prevalência de diabetes em grupos com condições de vida mais precárias e não beneficiários do bolsa família, reforçando a urgência de políticas sociais estruturantes para o enfrentamento de agravos crônicos.

Palavras-chave: Diabetes mellitus, Prevalência, Fatores de risco, Fatores socioeconômicos.

\begin{abstract}
Objective: To analyze the prevalence and socioeconomic determinants of diabetes mellitus in adults in the state of Pernambuco, Northeast Brazil, in 2015/2016. Methods: This is a cross-sectional study, with a sample of 1019 adults, based on the IV State Health and Nutrition Survey. A dependent variable was defined considering diabetics as those with fasting glucose $\geq 126 \mathrm{mg} / \mathrm{dl}$ or who would refer or be diagnosed during the interview. Possible associations between diabetes and socioeconomic determinants were analyzed using Poisson multivariate regression, considering as significant those with $p<0.05$. Results: The prevalence of diabetes was $10.8 \%$, being higher among the people with the lowest schooling (19\%), retired (23\%), who did not receive the family allowance (14.1\%) and who presented destination of leave $(15,9 \%)$ and water supply (15.8\%) offered irregularly. In the multivariate model as socioeconomic variables associated with diabetes were: schooling, occupation, family allowance and water supply. Conclusion: The study revealed a high prevalence of diabetes in groups with more precarious living conditions and not beneficiaries of the Bolsa Família, reinforcing the urgency of structuring social policies for coping with chronic conditions.
\end{abstract}

Keywords: Diabetes mellitus, Prevalence, Risk factors, Socioeconomic factors.

\footnotetext{
1 Instituto Aggeu Magalhães/Fundação Oswaldo Cruz (IAM/Fiocruz), Recife - PE.

*E-mail: silviaps2008@gmail.com

2 Universidade Federal de Pernambuco (UFPE), Recife - PE.
}

O estudo integra a pesquisa "IV Pesquisa Estadual de Saúde e Nutrição, em 2015/2016", a qual foi financiada pelo Conselho Nacional de Desenvolvimento Científico e Tecnológico (CNPq), $n^{\circ}$ 476862/2013-2 e Fundação de Amparo à Ciência e Tecnologia de PE (FACEPE), APQ-0025-4.00/2013. 


\section{RESUMEN}

Objetivo: Analizar la prevalencia y los determinantes socioeconómicos de la diabetes mellitus en adultos en el estado de Pernambuco, noreste de Brasil, en 2015/2016. Métodos: Se trata de un estudio transversal, con una muestra de 1019 adultos, con base en la IV Encuesta Estatal de Salud y Nutrición. La variable dependiente se definió considerando a los diabéticos con glucemia en ayunas $\geq 126 \mathrm{mg} / \mathrm{dl}$ o que remitieron su diagnóstico durante una entrevista. Las posibles asociaciones de diabetes con determinantes socioeconómicos se analizaron mediante regresión multivariante de Poisson, considerando significativas aquellas con $p<0,05$. Resultados: La prevalencia de diabetes fue del 10,8\%, siendo mayor entre las personas con menor nivel educativo (19\%), jubilados (23\%), que no recibían asignación familiar (14,1\%) y que tenían un destino de residuos $(15,9 \%)$ y suministro de agua $(15,8 \%)$ ofrecido de forma irregular. En el modelo multivariado, las variables socioeconómicas asociadas a la diabetes fueron: educación, ocupación, asignación familiar y suministro de agua. Conclusión: El estudio reveló una alta prevalencia de diabetes en grupos con condiciones de vida más precarias y no beneficiarios de Bolsa Família, lo que refuerza la urgencia de políticas sociales estructurales para enfrentar condiciones crónicas.

Palabras clave: Diabetes mellitus, Predominio, Factores de riesgo, Factores socioeconómicos.

\section{INTRODUÇÃO}

Parte da carga das Doenças Crônicas Não Transmissíveis (DCNT) resulta das condições em que as pessoas nascem, vivem, trabalham e envelhecem, ou seja, da atuação dos determinantes sociais sobre sua saúde. A influência desses determinantes na vida das pessoas as posiciona em diferentes contextos socioeconômicos, que por sua vez influência no maior ou menor acesso a recursos básicos, como os relacionados à promoção da saúde e prevenção de agravos (FRANCISCO PMSB, et al., 2015; CARRAPATO P, et al., 2017; WU H, et al., 2018).

Nesse contexto, destaca-se o diabetes mellitus (DM), um problema de saúde pública com notável variação em sua prevalência entre países, conforme os estudos de Santos J, et al. (2017) e Vonglokham M, et al. (2019).

De acordo com a World Health Organization (WHO) (2016) estima que a proporção de adultos acometidos por esse agravo quase duplicou em três décadas, passando de 4,7\% em 1980 para 8,5\% em 2014. Dados do Brasil também confirmam a tendência crescente na prevalência do diabetes, revelando um aumento de 24\% desde 2006 até 2018, sendo registrados 5,5\% em 2006 e 7,7 \% em 2018, significando altos custos para os serviços de saúde, uma vez que o DM gera grandes e subestimados efeitos econômicos adversos para as famílias, comunidades e sociedade em geral (SANTOS J, et al., 2017; BRASIL, 2019).

Ao contrário de muitos fatores de risco que se mostram consistentemente associados com o DM, como faixa etária, sobrepeso/obesidade e inatividade física, a associação entre fatores socioeconômicos e o problema não está bem estabelecida na literatura e difere entre as populações, segundo as pesquisas de Wu H, et al. (2017) e Wemrell M, et al. (2019).

Pesquisa realizada no Canadá, por exemplo, apontou que os últimos relatórios de saúde do país, sobre as causas associadas ao DM, têm focado naquelas modificáveis como o excesso de peso e o tabagismo, mas não têm analisado a possível relação com fatores socioeconômicos, como a renda. Assim, tal estudo ressalta que embora seja importante se concentrar nos fatores de risco modificáveis da doença, essa abordagem pode ser insuficiente se desconsiderar a natureza social e econômica do agravo em questão (BIRD Y, et al., 2015).

Um recente estudo realizado pela Fiocruz e outros centros de pesquisas demonstrou a associação entre a segregação residencial e condições relacionadas à saúde, incorporando o contexto de moradia à etiologia do diabetes. Assim, indivíduos que moram em vizinhanças mais segregadas economicamente locais com maior número de indivíduos responsáveis pelo domicílio com renda menor do que três salários mínimos; têm $50 \%$ mais chances de desenvolverem diabetes (BARBER S, et al., 2018).

A região Nordeste tem sua evolução histórica, durante o século $X X$, marcada pelo atraso econômico e social e pelas disparidades econômicas regionais. É inegável que nos últimos 50 anos, esta região teve 
crescimento significativo, contudo a questão das desigualdades intra e interregionais permanecem, mantendo-se em atraso relativo em relação ao Sul do país, pelo alto nível de pobreza que ainda perdura e continua sendo a região com desenvolvimento econômico mais desfavorável do país (ALBUQUERQUE MV, et al., 2017).

Diante da magnitude do DM no Brasil e no mundo, torna-se oportuno conhecer melhor os determinantes associados à sua ocorrência, particularmente em uma região do país onde esse objeto ainda é pouco explorado no campo da saúde coletiva. Portanto, faz-se necessário gerar conhecimento científico para tomada de decisões pelos gestores de saúde. Esse estudo foi desenvolvido no estado de Pernambuco, o segundo mais populoso da região nordeste e considerado um dos estados mais pobres do Brasil, marcado por desigualdades sociais como consta no INSTITUTO BRASILEIRO DE GEOGRAFIA E ESTATISTICA (IBGE) (2017) e objetivou analisar a prevalência e os determinantes socioeconômicos do diabetes mellitus em adultos com 20 anos e mais no estado de Pernambuco, nordeste brasileiro, em 2015/2016.

\section{MÉTODOS}

Foi realizado um estudo de corte transversal, de base populacional, utilizando dados da IV Pesquisa Estadual de Saúde e Nutrição, em 2015/2016 (IV PESN 2015/2016), no estado de Pernambuco, desenvolvida através de uma parceria entre a Universidade Federal de Pernambuco, o Instituto de Medicina Integral Professor Fernando Figueira, o Instituto Aggeu Magalhães, Fiocruz e a Secretaria de Saúde do Estado de Pernambuco. Esse inquérito foi proposto para atualizar e ampliar o diagnóstico de saúde, nutrição, alimentação, demandas de serviços e condições socioeconômicas dessa população.

Para o cálculo da amostra considerou-se uma prevalência de 13,0\% de diabetes em adultos com 20 anos e mais em Pernambuco, com base nos dados da III PESN realizado pela UFPE (2006), assumiu-se um erro de estimativa de $2,3 \%$ e um intervalo de confiança de $95 \%$, resultando numa amostra inicial de 821 adultos. Sobre este valor foi acrescentado $20 \%$ na amostra, para compensar possíveis perdas ou problema de não resposta, obtendo-se assim, uma amostra de 986 participantes. A amostra final, entretanto, contou com um total de 1019 adultos. Para este cálculo foi utilizado o software Epi Info versão 6.04 (Centers for Disease Control and Prevention, Atlanta, Estados Unidos).

O processo de amostragem desse inquérito foi do tipo probabilístico e estratificado em quatro estágios. Inicialmente foi realizado um sorteio dos municípios, em seguida dos setores censitários, posteriormente dos domicílios e, por fim, dos indivíduos. Tomou-se como base a seleção aleatória dos municípios na III PESN, totalizando 13 municípios, oito municípios da área rural (Vicência, São Bento do Una, Panelas, Caruaru, Serra Talhada, Custódia, Belém do São Francisco e Palmares) e cinco da área urbana (Recife, Paulista, Olinda, Cabo e Jaboatão). No segundo estágio, as unidades de seleção (setores censitários) também foram selecionadas randomicamente e sem reposição, utilizando registros do Censo Demográfico 2010.

Dessa forma, foram selecionados 20 setores censitários na zona urbana e nove na zona rural. No terceiro estágio, aproximadamente 40 domicílios foram selecionados com probabilidades iguais dentro de cada setor censitário. Para isso foi realizada uma seleção sistemática, com escolha aleatória de um ponto inicial. Em cada domicílio selecionado, um adulto foi sorteado a participar da entrevista e realizar exames referente a morbidades, como o de glicemia.

Os dados foram obtidos por meio de questionários padronizados e estruturados os quais continham informações de dados socioeconômicos, domiciliares, e referentes a morbidades. A aplicação dos questionários foi realizada por duas duplas de técnicos treinados. A coleta de dados foi realizada em domicílios de áreas urbanas e rurais do estado de Pernambuco, entre os meses de junho de 2015 a novembro de 2016.

A variável dependente do estudo foi o diabetes mellitus. Foram considerados casos de diabetes aqueles adultos com níveis de glicemia de jejum compatíveis com o seu diagnóstico, ou seja, $\geq 126 \mathrm{mg} / \mathrm{dL}$, parâmetro adotado pela American Diabetes Association (2013) ou que referiram o diagnóstico prévio do DM durante a entrevista. 
Para a determinação da glicemia de jejum foram coletadas amostras de sangue capilar, em jejum de 12 horas, usando-se o equipamento ACCUTREND GCT, de leitura imediata. A coleta sanguínea foi realizada no dia subsequente a entrevista, em domicílio. A equipe de laboratório foi composta por um supervisor e dois auxiliares de enfermagem com prática em atividades laboratoriais e foi realizado treinamentos específicos para utilização do aparelho ACCUTREND GCT. O diagnóstico referido do DM foi obtido a partir da pergunta: Algum médico, enfermeiro ou agente comunitário de saúde já lhe disse que o(a) sr.(a) tem diabetes?.

As variáveis independentes foram apresentadas de forma categórica, sendo consideradas como socioeconômicas: a) classe econômica: avaliada com base no critério da Associação Brasileira de Empresas de Pesquisa (ABEP) (2014), sendo definida a partir de um sistema de pontos que considera a posse de bens e o grau de instrução do chefe de família (B1/B2, C1/C2 e D/E); b) escolaridade (analfabeto/fundamental 1 incompleto, fundamental 1 completo/ fundamental 2 incompleto/completo, médio incompleto/completo/superior incompleto/completo); c) raça/etnia, informação autorreferida (branca e parda/preta/ outras), d) ocupação (empregado, autônomo e outros, aposentado, não trabalha/desempregado); e) recebe bolsa família (sim e não); f) habitação - área (rural e urbana), parede (tijolo/alvenaria e outros, teto (laje, telha e outros; g) saneamento básico - destino do lixo (coleta pública e outros, destino de dejetos (rede geral, fossa com tampa e outros e abastecimento de água (rede geral e outros).

Inicialmente foram realizadas análises descritivas para caracterizar a distribuição de frequência das variáveis. Posteriormente foram efetuadas análises bivariadas, através da Regressão de Poisson simples, para evidenciar possíveis associações de DM com as variáveis independentes. A categoria das variáveis que apresentou a melhor condição ou a menor prevalência do desfecho foi considerada como referência para as análises.

As variáveis que apresentaram nas análises bivariadas valor de $p<0,20$ foram selecionadas para ingressar na etapa seguinte de ajustamento (análise multivariada). As análises foram realizadas por meio de Regressão de Poisson, com ajuste robusto do erro padrão. Os resultados foram expressos por RP ajustadas com respectivos Intervalos de Confiança de 95\% (IC95\%) e foram retidos no modelo final aquelas variáveis com nível de significância $p<0,05$. As análises estatísticas foram processadas com 0 auxílio de software SPSS, versão 13.0 (SPSS Inc., Chicago, Estados Unidos) e Stata, versão 14.0 (Stata Corp., College Station, Estados Unidos).

A pesquisa primária (IV PESN) foi aprovada pelo Comitê de Ética em Pesquisa em Seres Humanos do Centro de Ciências da Saúde da Universidade Federal de Pernambuco (CAAE n. 07803512.9.0000.5208/ 2014, $n^{\circ}$. de protocolo do CEP 1.063.519), de acordo com os preceitos da Resolução do CNS 466/12. Os adultos investigados foram informados quanto a participação voluntária na pesquisa e assinaram o Termo de Consentimento Livre e Esclarecido (TCLE).

\section{RESULTADOS}

A prevalência do diabetes na população adulta avaliada no estado de Pernambuco foi de $10,8 \%$. Dos 1019 participantes do estudo, $70,6 \%$ residiam na zona urbana, 39,4\% foram classificados na classe econômica mais pobre (D/E), 39,4\% tinham menor grau de escolaridade (analfabetos/fundamental 1 incompleto), $74,3 \%$ eram da raça parda/preta e outras, $39,1 \%$ não trabalhavam, ou seja, estavam desempregados e 49,7\% recebiam o bolsa família. Quanto ao saneamento básico, destaca-se que apenas $43,8 \%$ contavam com rede geral para o destino de dejetos (Tabela 1).

Ao se analisar o DM de acordo com as variáveis socioeconômicas (Tabela 1), observou-se frequência semelhante entre as diferentes classes econômicas. As pessoas com menor grau de instrução (19\%), aposentados $(23 \%)$, que não recebiam bolsa família $(14,1 \%)$ e que apresentavam destino de dejetos $(15,9 \%)$ e abastecimento de água ofertado de outras formas (15,8\%) tiveram uma maior prevalência de DM. Observou-se também, que o problema era mais frequente entre os adultos que não apresentavam adequado destino de dejetos e de abastecimento de água. 
Tabela 1 - Prevalência e razão bruta do diabetes mellitus em adultos, segundo variáveis socioeconômicas. Pernambuco, 2015/2016.

\begin{tabular}{|c|c|c|c|c|c|c|}
\hline \multirow{2}{*}{ Variáveis } & \multicolumn{2}{|c|}{ Amostra } & \multicolumn{2}{|c|}{ Diabetes Mellitus } & \multirow{2}{*}{$\begin{array}{l}\text { RP bruta } \\
\text { (IC 95\%) }\end{array}$} & \multirow[b]{2}{*}{$p$} \\
\hline & $\mathbf{n}$ & $\%$ & & $\%$ & & \\
\hline Classe econômica & 1019 & & & & & 0,569 \\
\hline B1/ B2 (31/2- 7 SM$\left.^{a}\right)$ & 53 & 5,2 & 8 & 15,1 & 1 & \\
\hline C1/C2 (11/2-2 SM) & 565 & 55,4 & 59 & 10,4 & $0,69(0,35-1,37)$ & \\
\hline $\mathrm{D} / \mathrm{E}(1 \mathrm{SM})$ & 401 & 39,4 & 43 & 10,7 & $0,71(0,35-1,42)$ & \\
\hline Escolaridade & 1019 & & & & & $<0,001$ \\
\hline $\begin{array}{l}\text { Médio completo/superior } \\
\text { incompleto/completo }\end{array}$ & 285 & 28 & 11 & 3,9 & 1 & \\
\hline $\begin{array}{l}\text { Fundamental } 1 \text { completo/ } \\
\text { fundamental } 2 \\
\text { incompleto/completo/Médio } \\
\text { incompleto }\end{array}$ & 386 & 37,9 & 33 & 8,5 & $2,21(0,02-1,14)$ & \\
\hline Analfabeto/Fundamental 1 incompleto & 348 & 34,2 & 66 & 19 & $4,91(2,64-9,12)$ & \\
\hline Raça/etnia & 1019 & & & & & 0,529 \\
\hline Parda/preta e outras & 757 & 74,3 & 79 & 10,4 & 1 & \\
\hline Branca & 262 & 25,7 & 31 & 11,8 & $1,13(0,77-1,68)$ & \\
\hline Ocupação & 1019 & & & & & $<0,001$ \\
\hline Empregado & 163 & 16 & 8 & 4,9 & 1 & \\
\hline Autônomo e outros & 254 & 24,9 & 25 & 9,8 & $2,00(0,93-4,34)$ & \\
\hline Não trabalha/desempregado & 398 & 39,1 & 30 & 7,5 & $1,53(0,72-3,28)$ & \\
\hline Aposentado & 204 & 20 & 47 & 23 & $4,69(2,28-9,65)$ & \\
\hline Bolsa Família & $1017^{b}$ & & & & & 0,001 \\
\hline Sim & 505 & 49,7 & 38 & 7,5 & 1 & \\
\hline Não & 512 & 50,3 & 72 & 14,1 & $1,87(1,29-2,71)$ & \\
\hline \multicolumn{7}{|l|}{ Habitação } \\
\hline Área & $1018^{b}$ & & & & & 0,244 \\
\hline Rural & 299 & 24,9 & 27 & 9 & 1 & \\
\hline Urbana & 719 & 70,6 & 83 & 11,5 & $1,28(0,84-1,93)$ & \\
\hline Parede & 1019 & & & & & 0,425 \\
\hline Tijolo/alvenaria & 985 & 96,7 & 107 & 10,9 & 1 & \\
\hline Outros $^{c}$ & 34 & 3,3 & 3 & 8,8 & $0,72(0,33-1,59)$ & \\
\hline Teto & 1019 & & & & & 0,111 \\
\hline Laje & 183 & 18 & 26 & 14,2 & 1 & \\
\hline Telha & 655 & 64,3 & 61 & 9,3 & $0,65(0,43-1,01)$ & \\
\hline Outros $^{d}$ & 181 & 17,8 & 23 & 12,7 & $0,89(0,53-1,51)$ & \\
\hline \multicolumn{7}{|l|}{ Saneamento Básico } \\
\hline Destino do lixo & 1019 & & & & & 0,323 \\
\hline Coleta pública & 786 & 77,1 & 89 & 11,3 & 1 & \\
\hline Outros $^{\mathrm{e}}$ & 233 & 22,9 & 21 & 9 & $0,79(0,51-1,25)$ & \\
\hline Destino dos dejetos & $1008^{b}$ & & & & & 0,039 \\
\hline Rede geral & 442 & 43,8 & 48 & 10,9 & 1 & \\
\hline Fossa com tampa & 390 & 38,7 & 34 & 8,7 & $0,80(0,53-1,21)$ & \\
\hline Outros $^{f}$ & 176 & 17,5 & 28 & 15,9 & $1,46(0,95-2,26)$ & \\
\hline Abastecimento de água & 1019 & & & & & 0,015 \\
\hline Rede geral & 835 & 81,9 & 81 & 9,7 & 1 & \\
\hline Outros $^{g}$ & 184 & 18,1 & 29 & 15,8 & $1,62(1,10-2,41)$ & \\
\hline
\end{tabular}

Nota: RP: Razão de prevalência. IC95\%: Intervalo de Confiança = 95\%.a: SM - salário mínimo. b: As diferenças de valores amostrais em algumas variáveis se devem às perdas de observações, por conta de questionários incompletos e/ou por inconsistência de dados. c: parede - outros (taipa, papelão e outros tipos de materiais precários). d: teto - outros (Telha de amianto e outros tipos). e: destino de lixo - outros (enterrado, queimado, deixado em terreno baldio e outras formas). f: destino de dejetos - outros (fossa rudimentar sem tampa, curso de água e outras formas). g: abastecimento de água - outros (poço, cacimba, nascente, chafariz, cisterna de chuva e outras formas). Fonte: Melo SPSM, et al., 2021. 
Após ajuste dos fatores socioeconômicos associados, mediante análise multivariada (Tabela 2), permaneceram no modelo final quatro variáveis: escolaridade, ocupação, bolsa família e abastecimento de água, as quais se associaram estatisticamente ao desfecho em estudo $(p<0,05)$. Portanto, as pessoas com menor grau de escolaridade apresentaram uma probabilidade cerca de quatro vezes maior de ocorrência do $\mathrm{DM}(\mathrm{RP}=3,9$ e IC95: 2,00-7,61). Já os aposentados tiveram duas vezes mais chances de serem diabéticos $(R P=2,38$ e IC95: 1,11-5,07). Não receber bolsa família e não ter abastecimento vinculado à rede geral de água aumentou em quase duas vezes a probabilidade de apresentar o desfecho ( $R P=1,58$ elC95: 1,052,37, $\mathrm{RP}=1,64$ e IC95: 1,10-2,44, respectivamente).

Tabela 2 - Modelo final. Análise multivariada do diabetes mellitus em adultos. Pernambuco, 2015/2016.

\begin{tabular}{|c|c|c|c|}
\hline Variáveis & RP ajustada ${ }^{a}$ & IC 95\% & $p$ \\
\hline Escolaridade & & & $<0,001$ \\
\hline Médio completo/superior incompleto/completo & - & & \\
\hline $\begin{array}{l}\text { Fundamental } 1 \text { completo/ fundamental } 2 \\
\text { incompleto/completo/Médio incompleto }\end{array}$ & 2,26 & $1,13-4,53$ & \\
\hline Analfabeto/Fundamental 1 incompleto & 3,90 & $2,00-7,61$ & \\
\hline Ocupação & & & 0,028 \\
\hline Empregado & - & & \\
\hline Autônomo e outros & 1,55 & $0,72-3,35$ & \\
\hline Não trabalha/desempregado & 1,26 & $0,60-2,64$ & \\
\hline Aposentado & 2,38 & $1,11-5,07$ & \\
\hline Bolsa Família & & & 0,026 \\
\hline Sim & - & & \\
\hline Não & 1,58 & $1,05-2,37$ & \\
\hline Abastecimento de água & & & 0,015 \\
\hline Rede geral & - & - & \\
\hline Outros $^{b}$ & 1,64 & $1,10-2,44$ & \\
\hline
\end{tabular}

Nota: RP: Razão de prevalência IC95\%: Intervalo de Confiança = 95\%. a: As variáveis que apresentaram na análise bruta valor de $p<0,20$ foram selecionadas para ingressar nesta etapa de ajustamento, ou seja, escolaridade, ocupação, bolsa família, teto, destino de dejetos e abastecimento de água, permanecendo no modelo final apenas as variáveis estatisticamente significativas com $p$ valor $<0,05$. b: abastecimento de água - outros (poço, cacimba, nascente, chafariz, cisterna de chuva e outras formas).

Fonte: Melo SPSM, et al., 2021.

\section{DISCUSSÃO}

A expressiva proporção de diabéticos observada neste estudo reflete um quadro preocupante, tendo em vista o cenário marcante de desigualdades socioeconômicas vivido pela população em estudo. Uma pesquisa realizada numa área urbana de pobreza da região Nordeste, encontrou uma elevada prevalência de doenças crônicas, incluindo DM, 56,7\% (MELO SPSC, et al., 2019). A alta prevalência deste grupo de doenças nesta região pode ser reflexo de piores condições de vida e de acesso aos serviços de saúde para controle dos fatores de risco, determinando mortes mais precoces e maior incidência de sequelas. Estudo realizado no Canadá sobre fatores socioeconômicos e DM também enfatizou altas taxas de DCNT e DM entre populações vulneráveis (BIRD Y, et al., 2015).

Globalmente, a prevalência de diabetes está fortemente relacionada à posição socioeconômica dos indivíduos. Pesquisas de países desenvolvidos relatam uma associação inversa entre posição socioeconômica e prevalência da doença, enquanto o contrário foi encontrado em alguns países em desenvolvimento (QI Y, et al., 2019; WU H, et al., 2018).

A desigualdade socioeconômica é um fator independentemente relacionado ao aumento de DCNT, como - DM, em populações de baixa renda. Indivíduos com baixa condição socioeconômica podem ser mais vulneráveis a tais doenças por várias razões, incluindo estresse psicossocial, níveis mais elevados de comportamento de risco como sedentarismo e alto consumo de alimentos mais calóricos, ricos em açúcar e gorduras, condições de vida insalubres, precário acesso ao saneamento básico e serviços de saúde, além da oportunidade reduzida de prevenir complicações (BIRD Y, et al., 2015; EZEH A, et al., 2016). 
No presente estudo, a prevalência de DM (10,8\%; IC95\% 9,0-12,9) identificada foi semelhante à verificada em estudo realizado no Canadá que encontrou prevalência de $9,0 \%$ entre canadenses de baixa renda e nos Estados Unidos (12,1\%) com populações hispânicas e não hispânicas de 20 anos ou mais (BIRD Y, et al., 2015; CHENG YJ, et al., 2019).

Já no Brasil, estudo realizado no Maranhão e em Santa Catarina, encontraram uma prevalência menor de DM, 5,4\% e 4,2\%, respectivamente (GUIMARÃES RA, et al., 2018; TORTORELLA CCS, et al., 2017). Também se observou que o resultado foi maior que as estimativas para o Brasil, 7,7\% de acordo com dados do Sistema de Vigilância de Fatores de Risco e Proteção para Doenças Crônicas por Inquérito Telefônico em 2018, e 7,5\% de acordo com dados da Pesquisa Dimensões Sociais das Desigualdades em 2008 (PDSD) e 8,4\% de acordo com dados da Pesquisa Nacional de Saúde (PNS) em 2014/2015 (BRASIL, 2019 ; FLOR LS e CAMPOS MR, 2017; MALTA DC, et al., 2019). Vale ressaltar que este último estudo analisou a prevalências de DM, segundo diferentes critérios diagnósticos (critério laboratorial - hemoglobina glicada ou autorreferido ou uso de medicamentos) e constatou uma variação na prevalência de 6,6 a 9,4\%.

Conforme Malta DC, et al. (2019), a prevalência do DM tende a ser mais elevada quando são adotados critérios simultâneos, como a associação do critério laboratorial ou uso de medicamentos e do critério laboratorial ou autorreferido, um exemplo disso é a PNS 2014/2015, que encontrou diferentes prevalências dependendo do método usado e do uso simultâneo de técnicas.

Embora o método autorreferido seja validado e vastamente utilizado em virtude do baixo custo e por não ser invasivo, o seu uso isolado tende a levar a subnotificação de casos, uma vez que o DM é uma doença silenciosa e estimativas apontam que $46 \%$ dos diabéticos não sabem que estão com a doença, como afirma a American Diabetes Association (2013). Não obstante, vale ressaltar que as diferenças entre as características metodológicas (ano do levantamento, o tipo de questão utilizada para indicar/diagnosticar a presença da doença, processo de amostragem e de entrevista) das diferentes pesquisas populacionais referidas impossibilitam comparações diretas entre os valores de prevalência encontrados.

Ficou evidente, no presente estudo, a grande influência dos determinantes sociais no processo de adoecimento, pois constatou-se uma probabilidade cerca de quatro vezes maior de ocorrência do DM em pessoas com menor grau de escolaridade, corroborando com os achados de Wemrell M, et al. (2019) e Qi Y, et al. (2019).

Nesse contexto, a escolaridade coloca-se como um importante indicador socioeconômico e implica em riscos diferenciados no processo saúde-doença, especialmente devido ao ambiente vulnerável de vida, do menor acesso aos serviços de saúde e às práticas mais desfavoráveis de alimentação, atividade física, cuidados com o corpo e prevenção de doenças, aumentando assim o risco ao diabetes. Pessoas com menor nível de escolaridade e que vivem em localizações geográficas menos favorecidas, são desproporcionalmente afetadas por doenças crônicas, como o DM, como afirma Ezeh A, et al. (2016).

Observou-se em nosso estudo associação entre ocupação e o DM com maior probabilidade do desfecho entre a categoria de aposentados, resultado semelhante também foi encontrado no estudo realizado por Bird Y, et al. (2015) em populações adultas do Canadá e na pesquisa de Qi Y, et al. (2019) na Holanda e nos estudos de Zhou $M$, et al. (2015) e Zhang $H$, et al. (2013), na China. Tendo em vista que a aposentadoria se associa, em geral, à perda da função social produtiva em torno da qual se organizam as funções vitais, físicas e mentais, sua associação com a maior prevalência de DM guarda coerência com a epidemiologia da doença (BIRD Y, et al., 2015).

Neste sentido, cabe lembrar que a maior frequência do DM entre os aposentados, também pode estar associada ao avanço da idade, uma vez que as pessoas nessa fase de vida geralmente apresentam mais de 50 anos, e pode ser justificada pelas alterações inerentes ao processo de envelhecimento com diminuição da secreção de insulina, pela redução da atividade física e pela presença de hábitos alimentares pouco saudáveis nesse grupo, de acordo com estudo de Malta DC, et al. (2017). A associação do DM com o avanço da idade está bem estabelecida na literatura (GUIMARÃES RA, et al., 2018; MALTA DC, et al., 2019; VONGLOKHAM M, et al., 2019). 
Neste estudo, a prevalência de DM foi maior entre os adultos que não recebiam o benefício social do bolsa família. Resultado não observado nas pesquisas de Labrecque JA e Kaufman JS (2019) e Bernal RTI, et al. (2019) com beneficiários e não-beneficiários do programa, que observaram maior probabilidade de DM entre beneficiários.

Pesquisas realizadas na região sudeste e nordeste do Brasil por Sperandio N, et al. (2017) e em Pernambuco por Coelho PL e Melo ASSA (2017) observaram que os beneficiários do bolsa família apresentavam maior consumo de alimentos saudáveis quando comparados aos não beneficiários observaram que os beneficiários do bolsa família apresentavam maior consumo de alimentos saudáveis quando comparados aos não beneficiários e concluíram que existe um efeito positivo nas transferências de renda sobre a qualidade da dieta das famílias beneficiárias, mostrando assim a eficiência do benefício não só na melhora da alimentação dos indivíduos, mas na saúde e na quebra do ciclo intergeracional de pobreza no longo prazo.

O menor consumo desses alimentos saudáveis e substituição por alimentos calóricos, ricos em gordura e açúcar simples, pela população não beneficiária, pode os tornar mais vulneráveis às doenças crônicas, como o DM e tal achado pode explicar parcialmente a maior frequência do agravo nesse grupo de pessoas. Contudo, mais estudos devem ser realizados para maior compreensão dessa associação.

Quanto ao saneamento básico, observou que as pessoas que apresentaram outras formas de abastecimento de água (poço, cacimba, nascente, chafariz, cisterna de chuva e outras formas) ao invés de serem abastecidas pela rede geral, mostraram maior chance de serem diabéticas. Segundo a PNAD contínua 2018, o Nordeste é a segunda região com a menor taxa de abastecimento de água do Brasil (80,2\%), conforme dados do IBGE (2019), valor semelhante ao observado em nosso estudo para o estado de Pernambuco $(81,9 \%)$. Tal iniquidade no acesso ao saneamento básico contribui para a sua consolidação como um importante determinante das desigualdades em saúde. A falta ou a precariedade do acesso a água representa situação de risco que propicia o aumento da prevalência de doenças crônicas (EZEH A, et al., 2016).

O saneamento básico representa uma proxy das condições socioeconômicas de uma população, assim o baixo acesso a esse serviço essencial, ao tratamento de resíduos, a falta de planejamento urbano é, também, determinante das DCNT, como DM, por se traduzir como situações de grande vulnerabilidade a saúde da população (EZEH A, et al., 2016).

O delineamento transversal do presente estudo constitui uma limitação nas análises de associação entre as variáveis explicativas e o DM, por impossibilitar inferir relação de causalidade. A impossibilidade financeira de realizar o teste bioquímico de glicemia venosa de jejum, visando a confirmação dos casos de diabetes também foi considerado como limitação-

E outra limitação encontrada foi a escassez de pesquisas nacionais sobre determinantes socioeconômicos e o desfecho para comparabilidade do presente estudo, considerando assim a necessidade de avançar nas pesquisas para além dos determinantes biológicos e comportamentais já bem estabelecidos na literatura.

\section{CONCLUSÃO}

Este estudo revelou elevada prevalência de DM na população adulta de Pernambuco, sendo maior entre aqueles com baixa escolaridade, aposentados, não beneficiários do bolsa família e nos que citaram irregular abastecimento de água, demonstrando a influência da desigualdade social no processo saúde-doença. Portanto, ressalta-se a importância de focar nos determinantes sociais, priorizar a intersetorialidade na agenda de saúde, criar programas de benefício social para os mais vulneráveis, além da necessidade de realizar mais estudos para melhor entender os fatores socioeconômicos associados à doença, auxiliando, assim, no desenvolvimento de políticas públicas que possam reduzir a carga do DM, proporcionando maior equidade em saúde, especialmente nessa região que já é vulnerável pelo seu contexto econômico e social. 


\section{REFERÊNCIAS}

1. ALBUQUERQUE MV, et al. Desigualdades regionais na saúde: mudanças observadas no Brasil de 2000 a 2016 . Ciência \& Saúde Coletiva, 2017; 22(4):1055-1064.

2. AMERICAN DIABETES ASSOCIATION. Standards of Medical Care in Diabetes. Diabetes Care, 2013; 36(Suppl 1):11-66.

3. ASSOCIAÇÃO BRASILEIRA DE EMPRESAS DE PESQUISA (ABEP). Critério de classificação econômica Brasil. São Paulo: ABEP; 2014.

4. BARBER S, et al. At the Intersection of Place, Race, and Health in Brazil: Residential Segregation and Cardio-Metabolic Risk Factors in the Brazilian Longitudinal Study of Adult Health (ELSA-Brasil). Social Science \& Medicine, 2018; 199 : 67-76.

5. BERNAL RTI, et al. Indicadores de doenças crônicas não transmíssiveis em mulheres com idade reprodutiva, beneficiárias e não beneficiárias do Programa Bolsa Família. Revista Brasileira de Epidemiologia, 2019; 22(suppl 2): E190012.supl.2.

6. BIRD $\mathrm{Y}$, et al. The relationship between socioeconomic status/income and prevalence of diabetes and associated conditions: A cross-sectional population-based study in Saskatchewan, Canada. International Journal for Equity in Health, 2015;12:14-93.

7. BRASIL. Ministério da Saúde (MS). VIGITEL Brasil 2018: Vigilância de Fatores de Risco e Proteção para Doenças Crônicas por Inquérito Telefônico. Brasília: MS; 2019.

8. CARRAPATO P, et al. Determinante da saúde no Brasil: a procura da equidade na saúde. Saúde e Sociedade, 2017; 26(3):676-689.

9. CHENG YJ, et al. Prevalence of Diabetes by Race and Ethnicity in the United States, 2011-2016. Journal of the American Medical Association, 2019; 322(24): 2389-2398.

10. COELHO PL, MELO ASSA. Impacto do Programa "Bolsa Família" sobre a qualidade da dieta das famílias de Pernambuco no Brasil. Ciência \& Saúde Coletiva, 2017; 22(2):393-402.

11. EZEH A, et al. The history, geography, and sociology of slums and the health problems of people who live in slums. Lancet, 2016; doi: 10.1016/S0140-6736(16)31650-6.

12. FLOR LS, CAMPOS MR. Prevalência de diabetes mellitus e fatores associados na população adulta brasileira: evidências de um inquérito de base populacional. Revista Brasileira de Epidemiologia, 2017; 20(1): 16-29.

13. FRANCISCO PMSB, et al. Desigualdades sociodemográficas nos fatores de risco e proteção para doenças crônicas não transmissíveis: inquérito telefônico em Campinas, São Paulo. Epidemiologia e Serviços de Saúde, 2015; 24(1):7-18.

14. GUIMARÃES RA, et al. Epidemiology of Self-Reported Diabetes Mellitus in the State of Maranhão, Northeastern Brazil: Results of the National Health Survey, 2013. International Journal of Environmental Research and Public Health, 2018; 16(1). pii: E47.

15. INSTITUTO BRASILEIRO DE GEOGRAFIA E ESTATISTICA (IBGE). Pesquisa Nacional por Amostra de Domicílios Contínua - PNAD contínua. Rio de Janeiro: IBGE; 2017.

16. INSTITUTO BRASILEIRO DE GEOGRAFIA E ESTATISTICA (IBGE). Pesquisa nacional por amostra de domicílios contínua: 2018. Rio de Janeiro: IBGE; 2019.

17. LABRECQUE JA, KAUFMAN JS. Health profile differences between recipients and non-recipients of the Brazilian Income Transfer Program in a low-income population. Cadernos de Saúde Pública, 2019; 35(6): e00141218.

18. LYRA R, et al. Prevalência de diabetes melito e fatores associados em população urbana adulta de baixa escolaridade e renda do sertão nordestino brasileiro. Arquivos Brasileiros de Endocrinologia \& Metabologia, 2010; 54(6): 560-566.

19. MALTA DC, et al. Fatores associados ao diabetes autorreferido segundo a Pesquisa Nacional de Saúde, 2013. Revista de Saúde Pública, 2017; 51 Supl 1:12s.

20. MALTA DC, et al. Prevalência de diabetes mellitus determinada pela hemoglobina glicada na população adulta brasileira, Pesquisa Nacional de Saúde. Revista Brasileira de Epidemiologia, 2019; 22 (suppl 2): e190006.supl.2.

21. MELO SPSC, et al. Doenças crônicas não transmissíveis e fatores associados em adultos numa área urbana de pobreza do nordeste brasileiro. Ciência \& Saúde Coletiva, 2019; 24(8): 3159-3168.

22. QI Y, et al. Adulthood Socioeconomic Position and Type 2 Diabetes Mellitus-A Comparison of Education, Occupation, Income, and Material Deprivation: The Maastricht Study. International Journal of Environmental Research and Public Health, 2019; 16 (8). pii: E1435.

23. SANTOS J, et al.. Diabetes: desigualdades socioeconómicas na população portuguesa em 2014. Acta Médica Portuguesa, 2017;30(7-8):561-567.

24. SPERANDIO N, et al. Impacto do Programa Bolsa Família no consumo de alimentos: estudo comparativo das regiões Sudeste e Nordeste do Brasil. Ciência \& Saúde Coletiva, 2017; 22(6):1771-1780.

25. TORTORELLA CCS, et al. Tendência temporal da prevalência de hipertensão arterial sistêmica e diabetes mellitus entre adultos cadastrados no Sistema Único de Saúde em Florianópolis, Santa Catarina, 2004-2011 Epidemiologia e Serviços de Saúde, 2017; 26(3):469-480.

26. UNIVERSIDADE FEDERAL DE PERNAMBUCO (UFPE). Centro de Ciências da Saúde. Departamento de Nutrição. III Pesquisa estadual de saúde e nutrição: saúde, nutrição, alimentação, condições socioeconômicas e atenção à saúde no estado de Pernambuco, 2006. Recife: 2012.

27. VONGLOKHAM M, et al. Prevalence and social and health determinants of pre-diabetes and diabetes among adults in Laos: a cross-sectional national population-based survey, 2013. Tropical Medicine \& International Health, 2019; 24(1):6572.

28. WEMRELL M, et al. Understanding the complexity of socioeconomic disparities in type 2 diabetes risk: a study of 4.3 million people in Sweden. BMJ Open Diabetes Research \& Care, 2019; 7(1): e000749.

29. WORLD HEALTH ORGANIZATION (WHO). Informe mundial sobre la diabetes: resumen de orientación [Internet]. Geneva: WHO; 2016. [cited 2020 apri 27]. Available from: http://www.who.int/diabetes/global-report/es/.

30. WU H, et al. Socioeconomic status and prevalence of type 2 diabetes in mainland China, Hong Kong and Taiwan: a systematic review. Journal of Global Health, 2017; 7(1):011103.

31. WU H, et al. Socioeconomic status and self-reported, screen-detected and total diabetes prevalence in Chinese men and women in 2011-2012: a nationwide cross-sectional study. Journal of Global Health, 2018;8(2):020501.

32. ZHANG H, et al. Relation of socio-economic status to impaired fasting glucose and Type 2 diabetes: findings based on a large population-based cross-sectional study in Tianjin, China. Diabetic Medicine, 2013;30: e157- 62.

33. ZHOU M, et al. Geographical variation in diabetes prevalence and detection in china: multilevel spatial analysis of 98,058 adults. Diabetes Care, 2015; 38: 72-81. 